\title{
Referentes epistemológicos en la producción de conocimiento psicológico sobre violencia contra las mujeres
}

\author{
Epistemological references in the production of psychological \\ knowledge about violence against women
}

\author{
Adriana Rodríguez Fernández ${ }^{1}$
}

\begin{abstract}
RESUMEN
En este artículo se discuten los principales referentes epistemológicos, teóricos y metodológicos que han orientado la investigación sobre violencia contra las mujeres desarrollada en la carrera de Psicología de la Universidad de Costa Rica en el período 1994-2020. Se analizaron más de cuarenta tesis de grado y posgrado cuyo propósito fue la comprensión psicológica de la magnitud, dinámica e impacto de la violencia hacia las mujeres en el país. Los resultados apuntan a la influencia de la epistemología feminista en la concepción de los problemas de conocimiento y la relación sujeto-objeto de estudio, así como el posicionamiento ético-político de quien investiga.
\end{abstract}

Palabras clave: epistemología, investigación, psicología, violencia contra las mujeres.

\begin{abstract}
This article discusses the main epistemological, theoretical and methodological references that have guided the research on violence against women developed by Universidad de Costa Rica's Psychology School, in 19942020 period. There were analyzed more than forty undergraduate and graduate tesis in order to understand the psychological magnitude, dynamics and/or impact of violence against women in the country. Results point to the influence of the feminist epistemology in the conception of knowledge problems and the study relationship subject-object, as well as the ethical-political position of the researcher.
\end{abstract}

Key Words: epistemology, research, psychology, violence against women.

\footnotetext{
${ }^{1}$ Universidad de Costa Rica. San José, Costa Rica. Magíster. Profesora asociada de la Escuela de Psicología. Investigadora Centro de Investigación en Estudios de la Mujer. Correo electrónico:

adriana.rodriguezfernandez@ucr.ac.cr

DOI: $10.15517 /$ WL.V1612.48470

Recepción: 22/02/2021 Aceptación: 10/08/2021
} 


\section{Introducción}

El III Estado de los Derechos de las Mujeres en Costa Rica nos muestra que las mujeres continúan estando en condición de desventaja en todas las esferas de la vida social y constata, además, que "no existe un espacio o momento vital en el que las mujeres no sean vulnerables frente a la violencia en sus distintas manifestaciones, grados y escenarios..." (INAMU, 2019, p. 245). Es decir, no hay una edad, un contexto o un lugar seguro para ser mujer, ya que la violencia se convierte en una constante en sus vidas.

Bien sabemos que, es después de un largo camino, que la violencia contra las mujeres es reconocida como un problema social, una violación a los derechos humanos y un asunto de salud pública. A su vez, podemos afirmar que su abordaje como fenómeno psicosocial es relativamente reciente, pues hasta los años setenta la comprensión de la violencia contra las mujeres se había visto muy influenciada por modelos biológicos e instintivos acerca de la agresividad humana, en cuyo énfasis, tal como afirman Jayme y Sau (2004), predominaban el estudio psicológico de la esfera individual y el análisis de la conducta. Así, toda serie de metáforas orgánicas han sido utilizadas para explicar su origen, desde la llamada "naturaleza violenta", hasta la "enfermedad" y la "patología mental".

Es de reconocer que diversos planteamientos teóricos desde la sociología y la psicología social contribuyen a establecer la relación entre la violencia y el ambiente social, así como su carácter intencional y su dimensión instrumental. No obstante, son las teorías feministas las que posicionan que la violencia contra las mujeres posee una dinámica particular, ya que de acuerdo con la filósofa Ana De Miguel (2015), ésta "aun en medio de un universo de violencia, presenta claves específicas. Es decir, formas más específicas de legitimación, basadas no en su condición de personas sino de mujeres” (p.252).

Las teorías feministas nos han ofrecido un nuevo marco de interpretación para antiquísimas realidades como la violencia contra las mujeres y, sobre todo, desde los planteamientos del feminismo radical, la violencia deja de ser un suceso individual o problema relacional para encontrar su origen en el sistema patriarcal y definirse como una violencia estructural contra las mujeres.

Sin duda, lo anterior implicó además un giro epistemológico en la construcción de conocimiento en torno al fenómeno de la violencia contra las mujeres y los modos de 
acercarse científicamente a esta realidad, pues tal y como nos describe Celia Amorós, el testimonio de las mujeres sobrevivientes de violencia pasó de ser considerado una "anécdota", a adquirir valor como unidad de análisis:

"Las violaciones, de este modo, como los asesinatos de mujeres, no son anécdotas. Hasta hace poco ni éstas ni aquéllas se sumaban porque eran anécdotas: mejor dicho, se las consideraba anécdotas porque no se sumaban. Sólo pueden sumarse magnitudes homogéneas, y sólo se las considera tales a la luz de los conceptos de una teoría: es así como pasan de la anécdota a la categoría (Amorós, 2015, p.11).

(...) Las mujeres llevaron a cabo por su parte una elaboración reflexiva autónoma de su propia situación en los «grupos de concienciación» que constituyeron para intersubjetivizar sus experiencias, haciéndolas pasar de ese modo de la anécdota a la categoría" (Amorós, 2015, p.15).

De la mano con los feminismos y las teorías de género, podemos decir que en los últimos años la psicología también ha contribuido a visibilizar la magnitud e impacto de la violencia en las distintas dimensiones de la vida de las mujeres y sus subjetividades. Y propiamente en la Universidad de Costa Rica, de acuerdo con Rodríguez y Akoka (2019), ésta ha ocupado un lugar fundamental como problema de conocimiento en la investigación psicológica realizada a nivel de tesis de grado y posgrado; existiendo no sólo un aumento significativo en la cantidad de investigaciones realizadas desde mediados de los años noventa, sino una mayor amplitud a nivel de las manifestaciones y contextos relacionados con la violencia contra las mujeres, las adolescentes y las niñas.

A la luz de lo anterior, me propongo responder a la pregunta: ¿Cuáles han sido los principales referentes epistemológicos, teóricos y metodológicos de la investigación psicológica en materia de violencia contra las mujeres producida en la Escuela de Psicología de la Universidad de Costa Rica?

\section{Metodología}

Inicialmente resulta importante contextualizar que este trabajo es producto de una investigación documental que analizó veinte años de producción de conocimiento psicológico sobre la violencia contra las mujeres en la Universidad de Costa Rica. En una primera etapa, se realizó una caracterización de dicha producción investigativa, sus 
principales tendencias, aportes y desafíos ${ }^{2}$. Durante la segunda etapa, se propuso analizar a profundidad los referentes epistemológicos, teóricos y metodológicos presentes en las tesis de investigación publicadas en la Escuela de Psicología en el período 1994-2020.

Posterior a una exhaustiva búsqueda bibliográfica en las bases de datos del Sistema de Bibliotecas, Documentación e Información de la Universidad de Costa Rica (SIBDIUCR), se seleccionaron un total de 43 investigaciones a partir de los siguientes criterios: 1 . Investigaciones realizadas en la modalidad de tesis para optar al grado de licenciatura o maestría en Psicología. 2. Realizadas entre los años 1994 a 2020. 3. Dirigidas a construir conocimiento psicológico sobre la magnitud, dinámica o impacto de la violencia contra las mujeres y violencia de género contra niñas y adolescentes.

Cabe mencionar que para la selección de los estudios se partió de una concepción amplia de la violencia contra las mujeres, reconociendo sus diversas manifestaciones, ámbitos y contextos (Rodríguez y Akoka, 2019). De igual manera, se optó por incluir tesis que abordaron la violencia doméstica y sexual contra niñas y adolescentes.

Como siguiente paso, se llevó a cabo un análisis de contenido basado en las categorías indicadas en la Tabla 1:

Tabla 1: Categorías para el análisis de las investigaciones psicológicas en violencia contra las mujeres

1. Referentes epistemológicos:

- concepción sobre la construcción de conocimiento psicológico en violencia contra las mujeres, niñas y adolescentes;

- compresión de la relación sujeto-objeto de estudio;

- lugar de quién investiga y su posición frente al problema de conocimiento (neutralidad-objetividad/ subjetividad-implicancia).

2. Referentes teóricos: teoría general, teoría sustantiva o aportes teóricos específicos.

3. Referentes metodológicos: enfoque metodológico, técnicas para la recolección y análisis de la información, limitaciones metodológicas para responder preguntas de investigación.

4. Dilemas éticos: reflexiones críticas, cuestionamientos o interrogantes sobre el abordaje investigativo en la materia.

Fuente: elaboración propia

2 Al respecto puede revisarse el artículo: “A dos décadas de la Convención de Belem do Pará: Una caracterización de la investigación psicológica sobre la violencia contra las mujeres realizada en la Universidad de Costa Rica, período 1994-2016” en https://revistas.ucr.ac.cr/index.php/wimblu/article/view/39600/40160. 


\section{Resultados}

Como primer elemento del análisis realizado se precisó el objeto de estudio de cada una de las tesis incluidas en la muestra documental. Al respecto, una buena parte de éstas abordó como objeto de estudio la violencia doméstica en el contexto de relaciones de pareja, matrimonio y convivencia (Fernández 1996, Ramírez 1997 , González 2001, Cordero 2003, Loaiza 2008, Vega 2009, Pacheco 2009, Araya 2010, Vargas 2012, Sequeira y Vázquez 2015) y la violencia en relaciones de noviazgo (Murillo 1996, Incer 2009, Abarca 2010, Araya y Rivera 2012, Artavia y Carranza 2012, Castro 2012).

Otras tesis de investigación se centraron en el problema del abuso sexual infantil e incesto, tanto desde la perspectiva adulta como de personas menores de edad (Villalobos y Naranjo 1994, Cover 1995, Retana 1998, Calderón 2003, Chacón y Picado 2005, Diaz y Navarrete 2005, Molina y Moreira 2006) y la violencia hacia personas menores de edad en el ámbito doméstico y educativo (Alvarado y Saborío 1998, Rojas y García 2003). En una menor cantidad, se ubicaron tesis cuyo objeto fue la violencia doméstica desde la perspectiva del ofensor (Marín y Villalobos 1999, Calderón y Vargas 2004, Sandí y Venegas 2008) y la violencia sexual desde esta misma perspectiva (Arce y Gutiérrez 2003, Pisoni 2006)

En cuanto a la violencia en el contexto institucional y comunitario, se abordó como problema de conocimiento el hostigamiento sexual en el contexto de estudio y trabajo (Carvajal 2004, Marín 2012, Torres 1996, Torres 2002, Torres 2009), la explotación sexual comercial (Segura 1999, Castro y Chávez 2002, Alfaro y Rojas 2003, Claramunt 2005, Guzmán 2006, Rodríguez 2006). De manera más reciente, se ubicaron tesis centradas en el acoso sexual en espacios públicos (Mesén, 2019) y la violencia obstétrica (Hernández, 2020).

En adelante, se expondrán los hallazgos más relevantes del análisis de contenido llevado a cabo, no sin antes aclarar que, si bien lo epistemológico, lo teórico y metodológico son componentes que no pueden separarse o desligarse en la lógica de una investigación, sino que están articulados entre sí; para fines prácticos se retomará cada uno por separado en la presentación y discusión de los resultados. 


\subsection{Referentes epistemológicos}

¿Cómo se concibe el problema de estudio? ¿Cómo se construye conocimiento válido sobre dicho problema? ¿Cuál es la relación que establece la persona investigadora con el problema que se propone investigar? Todas estas preguntas están relacionadas con los fundamentos epistemológicos de una investigación. En ese sentido, tal como señala Blázquez (2012) "la epistemología es una teoría del conocimiento que considera lo que se puede conocer y cómo, o a través de qué pruebas las creencias son legitimadas como conocimiento verdadero. La epistemología feminista estudia lo anterior, abordando la manera en que el género influye en las concepciones del conocimiento, en la persona que conoce y en las prácticas de investigar, preguntar y justificar." (p.22)

¿Cómo se concibe la construcción de conocimiento psicológico sobre violencia contra las mujeres? Es característico en los diversos estudios analizados el planteamiento sobre la construcción de conocimiento a partir de la experiencia y la vivencia de las personas participantes (Murillo, 1996; Ramírez, 1997; Segura, 1999; González, 2001; Salas, 2002; Camacho, 2006; Blanco, 2006; Rodríguez, 2006; Sandí y Venegas, 2008; Loaiza, 2008; Incer, 2009; Torres, 2009; Abarca, 2010; Castro, 2012; Mesén, 2018; Hernández, 2019; entre otras) En ese sentido, se plantea como válido el conocimiento que se produce sobre la base de la profundización de los significados que las personas atribuyen a los fenómenos, en este caso a la violencia contra las mujeres. Predomina entonces, un interés por rescatar cómo los sujetos de estudio entienden e interpretan su propia realidad, qué valores y creencias están implicadas, “...para poder ver el mundo desde su propia forma de percibir el mundo y, de esa forma, tener un mejor acercamiento a sus experiencias." (Calderón y Vargas, 2004, p.2) Se parte, claro está, de que la realidad es construida socialmente: "el conocimiento se construye con los agentes sociales, en tanto que ellos son determinados y determinan el orden social en el que se inscriben y coloca como materia de estudio el propio discurso del sujeto con respecto a su vivencia subjetiva" (Sandí y Venegas, 2008, p.36)

Rescatar la vivencia subjetiva, explorar variantes de la subjetividad, partir de las representaciones y el sentido que las personas otorgan a su propia experiencia, son elementos que atraviesan el planteamiento epistemológico de la mayoría de las investigaciones 
(Fernández, 1996; Retana, 1998; Jiménez, 1998; Segura, 1999; Calderón y Vargas, 2004; Díaz y Navarrete, 2005; Rodríguez, 2006; Sandí y Venegas, 2008; Artavia y Carranza, 2012; Araya y Rivera, 2012; Castro, 2012; Mesén, 2019; Hernández, 2020; entre otras) Según señalan Díaz y Navarrete (2005) lo que las mujeres aportan, dicen y hacen se convierte en la parte central del análisis. Se reconoce así, una epistemología que implica una inmensa valoración de la singularidad "es la singularidad del sujeto la que se tiene en cuenta como revelador de una determinada vivencia social" (Pourtois y Desmet citado en Sandí y Venegas, 2008, p.155). Aunque se valida cada experiencia como única, también se propone rescatar lo común en la respuesta de las mujeres frente a la violencia (Salas, 2002; Alfaro y Rojas, 2003; Rodríguez, 2006; Incer, 2009; Mesén 2019; Hernández, 2020).

La construcción de conocimiento psicológico sobre este fenómeno se ha basado además en una visión retrospectiva, es decir, se propone una aproximación al problema de estudio mediante la experiencia de mujeres que fueron víctimas de diversas manifestaciones de violencia en el pasado (Castro y Chávez, 2002; Alfaro y Rojas, 2003; Araya, 2010) Es decir, el sentido histórico constituye un referente epistemológico a añadir.

De igual manera, la cotidianidad de las personas constituye un referente importante en la construcción de dicho conocimiento (Marín y Villalobos, 1999; Molina y Moreira, 2006; Rodríguez 2006, Mesén, 2020). El terreno de lo cotidiano se torna ineludible en esta pretensión de captar la realidad tal como la viven quienes participan del estudio, y comprenderles desde su propio marco de referencia (Torres, 2009; Araya y Rivera, 2012). Pero al mismo tiempo, se traduce en material de análisis necesario cuando se construye conocimiento desde los márgenes:

Dicha reflexión arroja una serie de cuestionamientos en torno a la aplicabilidad de estas premisas teóricas en contextos caracterizados por la exclusión social y la violencia extrema. Más allá de aplicar una teoría a esta realidad, se consideró importante partir de la realidad misma de las participantes, explorando y analizando sus vivencias específicas, con el fin de aportar soluciones y respuestas que se ajusten más a sus condiciones (Rodríguez, 2006, p.160).

Resulta evidente que en el plano epistemológico de los estudios predomina una relación de comprensión-descubrimiento en lugar de medición-comprobación; se trata más bien de un ejercicio investigativo inductivo que en palabras de Gurdián (citada en Artavia y 
Carranza, 2012) parte del hallazgo, sin ser su objetivo la comprobación o validación. Siendo así, que en la mayoría estos estudios se subraya la no pretensión de generalización de sus resultados, pero sí la profundización de la información y la riqueza interpretativa (Villalobos y Naranjo, 1994; Calderón y Navarro, 2003; Alfaro y Rojas, 2003; Molina y Moreira, 2006; Rodríguez, 2006; Vega, 2009; Araya, 2010; Araya y Rivera, 2012; Castro 2012, Hernández, 2020). Con una presencia menor, algunos estudios plantean su relación de conocimiento a través de la medición, en términos de incidencia o prevalencia de alguna manifestación de violencia (Cover, 1995; Carvajal, 2004; Marín, 2012), la correlación de variables o factores vinculados al fenómeno de estudio con interés predictivo (Chacón y Picado, 2005; Pisoni, 2006; Vargas, 2012), o bien, la comprobación de hipótesis concretas sobre causalidad (Pacheco, 2004; Sequeira y Vázquez, 2015)

No obstante, incluso aquellos estudios que se proponen medir, comprobar o relacionar factores vinculados con la ocurrencia de la violencia o el impacto de esta, plantean la dificultad -o el riesgo- de establecer relacionales causales tratándose de un fenómeno complejo y de múltiples dimensiones (Alvarado y Saborío, 1998; Vargas, 2012; Sequeira y Vázquez, 2015). De modo que, en la comprensión de la naturaleza misma del objeto de estudio se reconoce su complejidad, diversidad, variabilidad, y que, en tanto fenómeno social, no es susceptible ni reductible a la predicción o estandarización.

¿Cómo se comprende la relación sujeto-objeto de estudio? Considerando que la mayoría de los estudios tienen como principal referente la construcción de conocimiento a partir de la experiencia subjetiva e intersubjetiva de sujetas y sujetos concretos, la relación entre quien investiga y quien es investigada o investigado se ve atravesada por dos elementos principales: la interacción y la horizontalidad. En ese sentido, algunos estudios plantean la relación dinámica que se establece entre ambos actores, la mutua influencia tanto en la captación de información como en el análisis e interpretación de la misma (Murillo, 1996; Segura, 1999; Rodríguez, 2006; Calderón y Navarro, 2003). Unido a ello, como punto de partida se establece que las personas investigadoras poseen un bagaje cultural intersubjetivo que es compartido durante el proceso de investigación, de acuerdo con Segura (1999) "La construcción del conocimiento se produce entonces como parte de un proceso en el que sujeto y objeto pueden enriquecerse mutuamente, aunque cada uno desde su lugar particular. La 
comprensión de los significados específicos es esencial, procurando ubicarlos como parte de la trama social más amplia que los acuerda" (p.82)

Partir de ese lugar epistemológico, en una buena parte de las investigaciones, implicó ir más allá de la mera observación o registro de datos, buscando la mayor interacción posible con las y los sujetos de estudio a través del diálogo (Calderón y Navarro, 2003; Salas, 2002; Rodríguez, 2006; Hernández, 2020) Así, se concibe la construcción de conocimiento como el resultado de un proceso dialógico donde la horizontalidad se torna necesaria e implica a su vez, reconocer a las participantes como sujetas de un saber. Como lo ejemplifica Cordero, su aproximación investigativa "propicia un espacio para que las verdaderas expertas sobre el tema, las mismas mujeres que han vivido esta experiencia, diluciden su vivencia y brinden sus aportes" (2003, p.2).

Si bien no todas las investigaciones analizadas se enmarcan propiamente desde una "epistemología feminista", puede interpretarse que muchos de sus planteamientos acerca de su relación con la violencia como problema de estudio constituyen referentes de las epistemologías feministas.

La investigación feminista muestra que las interacciones en las que las mujeres son más libres para crear con otros significados que describen sus experiencias vitales son las que se producen en las relaciones y comunicaciones con mujeres situadas en posiciones similares (Lenermann y Brantley, citado en Salas, 2002, p.402).

¿Cuál es el lugar de quien investiga? ¿Qué posición se asume frente al objeto de estudio? A nivel general, el análisis realizado permitió dilucidar que los estudios psicológicos sobre violencia contra las mujeres se alejan de esa "neutralidad objetiva" heredada por la tradición positivista en la ciencia, para acercarse a una relación de mayor proximidad e implicancia con respecto a su objeto de estudio. Lo anterior se ve plasmado de distintas formas. Por un lado, algunas tesis hacen referencia al interés personal de quien investiga (Fernández, 1996; Abarca, 2010, Mesén, 2020) o su compromiso ético-político frente a la problemática:

No se pretende entonces asumir un papel neutral con relación a la problemática de estudio, más bien, a partir de que ambas partes, investigadora 
y población de estudio, son producto de un mismo contexto vivencial, que se refleja en su construcción y características particulares (Segura, 1999, p.82)

Por otra parte, en ciertos estudios se da un lugar a la propia subjetividad de quien investiga como un elemento más para ser interpretado o analizado (Villalobos y Naranjo, 1994; Ramírez, 1997; Segura, 1999). En esta misma línea, con relación al problema de la violencia, algunas investigadoras hacen referencia a su lugar como mujeres, marcando desde allí, su implicancia o afectación frente a la realidad que intentan desentrañar (Mesén, 2019; Hernández, 2020). Al respecto, cabe agregar que la mayoría de las tesis analizadas fueron elaboradas por mujeres. De acuerdo con Rodríguez y Akoka (2019) del total de investigaciones psicológicas en materia de violencia producidas en la Universidad de Costa Rica entre los años 1994 a 2016, casi el 93\% fueron realizadas por mujeres.

Es valioso mencionar además que algunas investigadoras analizan directamente los efectos que les produce su acercamiento al problema de estudio y su exposición a los testimonios de las participantes. Tal es el caso de Segura, quien se refiere a su relación contratransferencial con las participantes: "había sentido mi propio cuerpo irrespetado con la sola mirada de las circunstancias que rodean la vida de todas las jóvenes (...) mi cuerpo reaccionó a la violencia que presenciaba" (1999, p.242).

¿Y lo ético en la investigación? ¿ ¿Para qué se investiga? Este aspecto se ve intrínsecamente relacionado con la posición que se asume frente a la violencia como problema de conocimiento psicológico. Ya se ha señalado antes que una cantidad significativa de investigaciones parten de $-\mathrm{o}$ al menos sugieren- un compromiso social (Fernández, 1996; Salas, 2002; Calderón y Vargas, 2004; Rodríguez, 2006; Castro, 2012; Marín, 2012; Sequeira y Vázquez, 2015; Mesén, 2010; Hernández, 2020). Dicho compromiso se traduce no sólo en tipo de acercamiento investigativo que se realiza, sino en los fines o aportes que se aspira alcanzar a través de este. Se parte de que toda práctica científica debe tener compromisos éticos con los valores de verdad, justicia y libertad (Salas, 2002) y planteándose que "desde el sector académico es esencial la contribución para el diseño de planes de monitoreo y exigibilidad de las competencias institucionales y compromiso" (Rodríguez, 2006, p.179)

De tal manera que, buena parte de los estudios se proponen ofrecer conocimiento útil para el cambio social, o bien, la mejoría de la respuesta institucional frente a la problemática 
de la violencia hacia las mujeres, las adolescentes y las niñas. Muestra de ello, que a partir de sus resultados se proporcionen guías, modelos, protocolos o incluso metodologías replicables (Loaiza, 2008; Vega, 2009; Abarca, 2010; Castro, 2012). Por su parte, algunas investigaciones señalan cómo el proceso metodológico en sí mismo implicó benefícios para las personas participantes, generando espacios para compartir sus experiencias y reflexionar sobre estas, tomar mayor conciencia sobre su situación de vida y ser escuchadas (Murillo, 1996; Calderón y Navarro, 2003; Molina y Moreira, 2006; Abarca 2010).

Lo anterior viene a representar otro referente más de las epistemologías feministas, las cuales no están desvinculadas de la noción de transformación social (Rodríguez e Ibarra 2013), pues esta representa más bien su origen o punto de partida.

"Es a partir de ese conocimiento que se enriquece la práctica psicológica, pues en la medida en que se establezca un acercamiento y se conozcan las vivencias de estas mujeres, se podrá entender y desarrollar prácticas terapéuticas que respondan a las necesidades reales de las personas y se constituyan en herramientas efectivas para su crecimiento personal"' (Chacón y Picado, 2005, p.15)

\subsection{Referentes teóricos}

En armonía con los referentes epistemológicos antes puntualizados, los planteamientos teóricos de las investigaciones se basan sobre todo en perspectivas que enfocan la violencia como producto del aprendizaje social en el sistema patriarcal. Esto se entiende a partir de una nueva interpretación de la violencia gestada en los años setenta, la cual supera el viejo determinismo genético-evolutivo para dar lugar a una comprensión de los factores sociales y culturales implicados (Loaiza, 2008). En este giro el surgimiento de la categoría género, fuera del terreno de lo biológico, busca desentrañar los significados y códigos socioculturales en la construcción de las masculinidades y feminidades.

De manera general, la mayoría de las tesis cimentan sus bases teóricas en troncos comunes: perspectivas psicosociales en torno a la violencia, los estudios de género y las teorías feministas, que hasta cierto punto, establecen diálogos y tienden puentes en la generación de conocimiento en materia de violencia (Fernández, 1996; Murillo, 1996; Salas, 
1996; Ramírez, 1997; Segura, 1999; Marín y Villalobos, 1999; Castro y Chávez, 2002; Salas, 2002; Rojas y García, 2003; Cordero, 2003; Calderón y Navarro, 2003; Alfaro y Rojas, 2003; Calderón y Vargas, 2004; Pacheco, 2004; Díaz y Navarrete, 2005; Camacho, 2006; Blanco, 2006; Molina y Moreira, 2006; Rodríguez, 2006; Sandí y Venegas, 2008; Loaiza, 2008; Incer, 2009; Torres, 2009; Vega, 2009; Araya, 2010; Abarca, 2010; Artavia y Carranza, 2012; Araya y Rivera, 2012; Castro 2012; Marín, 2012; Vargas, 2012; Sequeira y Vázquez, 2015; Mesén, 2019; Hernández, 2020)

Se parte además de teorías que incorporan las subjetividades, por ejemplo, como afirma Salas (2002) “para explicar el fenómeno del acoso sexual es necesario ubicarse desde una perspectiva teórica que ofrezca la posibilidad de incorporar lo subjetivo" (p.37) De acuerdo con Camacho (2006) la teoría de género toma en cuenta la influencia de las experiencias personales y colectivas, así como de las relaciones de poder instauradas a partir de los sistemas sociales, políticos y culturales.

Puede afirmarse entonces, que existe una mayor tendencia a plantear estudios desde teorías de género, que en esencia ponen en cuestión los postulados sobre el origen de la subordinación femenina, comprendiendo las diferencias de género en la dimensión de la desigualdad y las relaciones de poder: "La teoría de género, como otra forma de interpretación, viene a desarrollar una mirada más atenta de la condición femenina en la cultura patriarcal y de su situación de subordinación sexual" (Camacho, 2006, p.14). Para Loaiza (1998, p.67) “Desde esta perspectiva la violencia contra las mujeres deja de ser natural y espontánea, como señalan algunas corrientes, y se convierte en un producto social derivado de las estructuras de poder desigual entre géneros". Dicha comprensión del origen social de la violencia contra las mujeres implica una ruptura con posicionamientos psicologistas que permearon en algún momento el desarrollo teórico en torno al fenómeno en cuestión.

El sentido histórico es además característico en las teorías de género y feministas, cuyo análisis parte de los procesos históricos en la construcción de las múltiples subjetividades femeninas y masculinas. De acuerdo con Camacho (2006) la teoría de género tiene como sujeto del conocimiento al individuo histórico particular cuyo cuerpo, intereses, emociones y razón están atravesados por su contexto histórico concreto.

Otro referente teórico, quizás en una línea aún más cercana a la Psicología, se refiere a los notables aportes de las teorías del trauma en la comprensión del impacto de las diversas 
manifestaciones de violencia, en especial la violencia por parte de la pareja y la violencia sexual, en un número significativo de estudios a lo largo del tiempo (Villalobos y Naranjo, 1994; Cover, 1995; Retana, 1998; Alvarado y Saborío, 1998; Marín y Villalobos 1999; González, 2001; Díaz y Navarrete, 2005; Chacón y Picado, 2005; Camacho, 2006; Araya, 2010). De acuerdo con Claramunt (1997) la teoría del trauma parte del reconocimiento de la influencia de factores externos, tales como eventos o situaciones altamente estresantes que debido a su naturaleza e intensidad ocasionan cambios en la manera de pensar, sentir y actuar de las personas que los experimentan. Este desarrollo teórico ha permitido profundizar en las implicaciones de la violencia en la vida de las mujeres y particularmente explicar las consecuencias psicológicas de dicha experiencia. Cabe señalar que en las investigaciones mencionadas se rescatan planteamientos teóricos de autores y autoras como Ronald Summit, Susan Blume, David Finkelhor y Angela Browne, Sara Sharrat, Ellen Bass y Laura Davis, Judith Hermann, Gioconda Batres.

Unido a lo anterior, es destacable el aporte de Leonor Walker $(1979,1990)$ en esta lectura psicológica de la violencia, siendo frecuente la referencia a su teoría del ciclo de la violencia contra las mujeres en bastantes de las investigaciones en las últimas dos décadas (Fernández, 1996; Jiménez, 1996; Ramírez, 1997; Cordero, 2003; Vega, 2009, entre otras).

En menor medida se identifican otros aportes desde teorías psicoanalíticas (Jiménez, 1998; Sandí y Venegas, 2008) y teorías cognitivo-conductuales (Chacón y Picado, 2005; Vargas, 2012; Sequeira y Vázquez, 2015), desde las cuales se busca dialogar con perspectivas de género. Otras teorías sustantivas incluidas en los estudios son la teoría del apego (Artavia y Carranza, 2012) y la teoría de representaciones sociales (Molina y Moreira, 2006; Segura, 1999). A su vez, se realizan planteamientos desde la psicología evolucionaria (Pacheco, 2004); la psicología forense (Arce y Gutiérrez, 2003; Pisoni, 2006); la psicología política (Mesén, 2019; Hernández, 2020); el enfoque de salud pública (Loaiza, 2008; Vega, 2009; Araya, 2010) así como, otros modelos teóricos particulares en la comprensión de la violencia tales como el modelo contextual generacional (Castro y Chávez, 2002) y el modelo ecológico (Araya, 2010).

A nivel general, un elemento que sobresale es la referencia a autoras y autores predominantemente latinoamericanos, y también de origen costarricense, lo cual da cuenta de un interés por el desarrollo de un conocimiento psicológico crítico y situado, que busca 
alejarse de la tradición euronorcentrista que ha permeado históricamente la ciencia psicológica. Como plantea Blanco (2006) al referirse al marco teórico de su estudio "representa un esfuerzo por no copiar irreflexivamente modelos importados que han surgido de realidades socioculturales e históricas distintas, sino crear una psicología desde Latinoamérica, que desde aquí plantee soluciones y líneas de acción” (p.260)

Resulta además muy frecuente la reseña de autores y autoras nacionales que han alimentado las perspectivas psicosociales en torno a la violencia hacia las mujeres, como Cecilia Claramunt, Mirta González, Laura Guzmán, Teresita Ramellini, Montserrat Sagot, José Manuel Salas y Gioconda Batres, entre otros.

Por otra parte, justamente la dimensión ética de la investigación en la materia conlleva a incluir planteamientos relacionados con la teoría de derechos humanos, los cuales se distinguen de manera más precisa en los estudios desarrollados a partir del año 1997 (Ramírez, 1997; Rodríguez, 1996; Blanco, 1996; Alfaro y Rojas, 2003; Carvajal 2004; Chacón y Picado, 2005; Claramunt, 2005; Blanco, 2006; Loaiza, 2008; Torres, 2009; Vega, 2009; Castro, 2012; Marín, 2012; Hernández, 2020) Algunos de estos estudios introducen aportes legales dentro de sus marcos de referencia teórico-conceptuales. En ese sentido, se enfatiza en definiciones jurídicas de violencia doméstica, violencia contra las mujeres y violencia basada en género, derivadas de instrumentos normativos nacionales e internacionales. Dichas definiciones son usadas tanto para la argumentación de la pertinencia social de la investigación, como para el análisis propio del fenómeno de estudio. Tal como sugiere Crempien “el cómo nombremos un fenómeno tendrá repercusiones en cómo lo vemos, cómo lo entendemos, qué sentido le damos y de qué manera lo enfrentamos como problema" (citada en Araya 2010, p.10).

No cabe duda de que en el plano de la incidencia política es fundamental la referencia a elementos normativos en materia de derechos humanos, sin embargo, desde una lectura crítica del cuerpo investigativo analizado, se identifica el riesgo de que "glosarios jurídicos"3 sustituyan la profundización en la discusión teórica sobre la violencia contra las mujeres como objeto de estudio de la ciencia psicológica.

\footnotetext{
${ }^{3}$ Término usado por la investigadora
} 


\subsection{Referentes metodológicos}

Como ya han planteado Rodríguez y Akoka (2019), en la investigación universitaria sobre violencia contra las mujeres desarrollada en las últimas décadas hay una predominancia de enfoques metodológicos de tipo cualitativo. Coherente con algunos de los referentes epistemológicos mencionados, la investigación cualitativa se posiciona como una alternativa de abordaje científico valiosa en el tanto permite aplicar un método que valora las subjetividades, buscando rescatar de la forma más natural posible la construcción de sentido de las personas participantes (Salas, 2002). Según plantea la autora con respecto a su propio estudio "Los significados psicosociales, por su naturaleza subjetiva, escapan a los métodos de las ciencias "duras". En cambio, la investigación cualitativa permitió el análisis y la interpretación del discurso mediante el cual se reveló el mundo subjetivo" (Salas, 2002, p.45)

De acuerdo con Camacho (2006), el enfoque cualitativo permite incorporar la introspección y la comprensión personal, así como las apreciaciones subjetivas en torno a acontecimientos de la historia individual; un rasgo característico en los planteamientos metodológicos de los estudios previos. Donde, además, el testimonio de las mujeres y niñas víctimas- sobrevivientes de violencia representa la principal fuente de información. Tal como lo indica (Araya, 2010), el relato de las mujeres como la única fuente para entender sus experiencias.

Lo anterior despierta preocupaciones éticas en algunas investigadoras, pues en ciertos casos la participación voluntaria de las mujeres, niñas o adolescentes se torna dilemática. Según lo señalan Camacho (2006) y Villalobos y Naranjo (1994) para algunas mujeres es incómodo compartir su historia con personas ajenas; el contar su historia puede generar temor o vergüenza. Lo cierto es que este tipo de dilemas éticos son mencionados en pocos de los estudios y de manera tangencial, es decir, no se profundiza lo suficiente en esta problematización. Algunas autoras advierten sobre la necesidad de que las participantes estén siendo atendidas y acompañadas psicológicamente durante el desarrollo en el estudio, aspecto que no siempre puede garantizarse.

En otro plano, los procesos de recolección de información desde las metodologías implementadas, se nutre de diversas técnicas de investigación, las cuales suelen además combinarse con el fin de alcanzar algunos criterios de confiabilidad y validez. Entre estas 
predomina el uso de la entrevista, tanto la estructurada como la entrevista en profundidad (Fernández, 1996; Ramírez, 1997; Retana, 1998; Marín y Villalobos, 1999; Cordero, 2003; Calderón y Navarro, 2003; Alfaro y Rojas, 2003; Calderón y Vargas, 2004; Blanco, 2006; Rodríguez, 2006; Incer, 2009; Torres, 2009; Vega, 2009; Araya, 2010; Araya y Rivera, 2012; Mesén, 2019). Al respecto, se defienden las virtudes de la entrevista como recurso metodológico que permite acceder a la construcción de sentido de las personas participantes, reconocer sus motivaciones, percepciones y discursos.

El estudio de caso se desarrolla como método en algunas de las investigaciones (Alvarado y Saborío, 1998; Segura, 1999; Marín y Villalobos, 1999; González, 2001; Camacho, 2006; Castro, 2012; Vargas, 2012) así como el método biográfico basado en historias de vida (Villalobos y Naranjo, 1994; Castro y Chávez, 2002; Artavia y Carranza, 2012) o los relatos de vida (Sandí y Venegas, 2008; Hernández 2020).

Adicionalmente, se aplican técnicas de recolección de información como la entrevista grupal (Díaz y Navarrete, 2005); grupos focales o grupos de discusión (Molina y Moreira, 2006; Rodríguez, 2006; Sandí y Venegas, 2008; Vega, 2009; Abarca, 2010; Araya y Rivera, 2012; Castro, 2012; Mesén, 2019); los talleres (Murillo, 1996; Salas, 1996; Salas, 2002; Rojas y García, 2003; Loaiza, 2008); el cuestionario o la encuesta (Pacheco, 2004; Carvajal 2004; Chacón y Picado, 2005; Blanco, 2006; Marín, 2012); la aplicación de pruebas o instrumentos estandarizados (Sequeira y Vázquez, 2015); y la revisión documental de expedientes judiciales (Arce y Gutiérrez, 2003; Pisoni, 2006)

Un referente común se refiere al desarrollo de procesos metodológicos que privilegian la interacción y las modalidades participativas que, desde el punto de vista de las investigadoras, propicia la construcción conjunta de conocimiento, permite observar la interacción entre participantes, al mismo tiempo que implica procesos de reflexión y retroalimentación, toma de consciencia y capacitación para el fortalecimiento de las participantes (Loaiza, 2008; Araya y Rivera, 2012). Al respecto se plantea:

Estos espacios significaron la apropiación de la palabra. De acuerdo con ellas, al interno de la entrevista enfocada y la actividad grupal, pudieron llevar a cabo una revisión de la historia, tomando en cuenta su pasado y su historia de abuso sexual, también se colaboró para que reconceptualizaran sus 
concepciones, nociones y percepciones acerca de la sexualidad, prácticas sexuales y disfrute de esta. (Díaz y Navarrete, 2005, p.196)

Los y las participantes pudieron expresar abiertamente sus opiniones, las que se convirtieron en discusiones enriquecedoras alrededor del tema de estudio (Rojas y García, 2003, p.49)

Finalmente, es importante añadir que en términos generales ha existido muy poca reflexión sobre limitaciones en el plano metodológico de los estudios, lo mismo que la profundización en torno a los dilemas éticos que conlleva este campo de investigación.

\section{Consideraciones finales}

Los resultados expuestos en este trabajo ponen el acento en la influencia que las epistemologías feministas han tenido en la producción de conocimiento psicológico sobre la violencia contra las mujeres, a nivel de tesis de grado y posgrado de la Universidad de Costa Rica. Hemos señalado que, si bien no todas las investigaciones se plantean desde un enfoque feminista determinado, en su mayoría poseen elementos característicos de una comprensión feminista no sólo del fenómeno de la violencia, sino de la relación de la persona investigadora con su objeto de estudio y su nivel de implicancia.

El análisis realizado deja entrever que la violencia, como objeto de estudio psicológico, se concibe como un fenómeno complejo, dinámico y cambiante, producto de condiciones históricas, sociales y culturales. En este marco, los referentes epistemológicos de las investigaciones revisadas se caracterizan por privilegiar las vivencias, los significados, los sentidos y las experiencias en la construcción de conocimientos, profundizando en ejes como la subjetividad y la cotidianidad.

Otros aspectos anclados a las epistemologías feministas están relacionados con la historización, la producción de un conocimiento situado, la noción de transformación y el compromiso ético-político en la investigación. Las rutas investigativas se definen no sólo por preguntas necesarias, sino por la aspiración de una sociedad basada en la igualdad y la justicia para las mujeres y las niñas. Podríamos decir que, hasta cierto punto, se trata de una investigación contracultural, cuestionadora del orden establecido y de las relaciones de 
poder. La reflexión sobre el poder se plasma no sólo en la comprensión misma de la violencia como problema social, sino en la relación entre la persona investigadora y quienes participan del proceso investigativo, que, a su vez, se conciben desde un lugar activo.

Finalmente, se reconoce la necesidad de una mayor reflexión en cuanto a los obstáculos epistemológicos, las limitaciones metodológicas y los dilemas éticos en este campo investigativo.

\section{Referencias bibliográficas}

Abarca Herrera, Amalia. "La violencia de género en relaciones de noviazgo en estudiantes del programa de residencia estudiantil de la sede de Guanacaste". Tesis de licenciatura. Universidad de Costa Rica, Guanacaste, C.R. 2010.

Alfaro, Francia y Rojas, Erika. "Explotación sexual comercial: una visión retrospectiva desde la experiencia de mujeres adultas trabajadoras sexuales, víctimas de explotación sexual comercial en la niñez o adolescencia”. Tesis de licenciatura. Universidad de Costa Rica, San José, C.R. 2003.

Alvarado, Rebeca y Saborío, Carlos. "Depresión infantil y violencia doméstica. Estudio realizado con niños y niñas en edad preescolar y víctimas de diferentes tipos de abuso intrafamiliar“. Tesis de licenciatura. Universidad de Costa Rica, San José. C.R. 1998.

Amorós, Puente Celia. 2005. "Dimensiones del poder en la teoría feminista”. Revista Internacional de Filosofía Política, 25 Recuperado de http://repositorio.ciem.ucr.ac.cr/jspui/bitstream/123456789/299/1/RCIEM264.pdf

Araya, Yessica. "Vivencia y construcción de una nueva vida de pareja en mujeres sobrevivientes de violencia doméstica". Tesis de licenciatura. Universidad de Costa Rica, San José, C.R. 2010.

Araya Brenes, Nancy y Rivera Ramírez, Vivian. "El papel de la violencia en el noviazgo adolescente y la conformación de la identidad masculina, desde la perspectiva de estudiantes varones de un colegio rural". Tesis de licenciatura. Universidad de Costa Rica, San José, C.R. 2012.

Arce, Benilda y Gutiérrez, Carolina. "Psicopatía en violadores y su forma de operar en el delito. Descripción acerca de los niveles de psicopatía en los distintos tipos de 


\section{वरिज्य}

Wimblu, Rev. Estud. de Psicología UCR, 16(2) 2021 (Julio-Diciembre): 137-159 /ISSN: 1659-2107

violadores de mujeres y su relación en la forma en cómo cometen el delito". Tesis de licenciatura. Universidad de Costa Rica, San José, C.R. 2003.

Artavia, Cindy y Carranza, Marco. "Estilos de apego en mujeres que permanecieron expuestas a situaciones de violencia en sus relaciones de noviazgo durante la adolescencia tardía”. Tesis de licenciatura. Universidad de Costa Rica, San José, C.R. 2012.

Blanco Guzmán, Nadia. "Explotación sexual comercial: dinámica de las familias de niñas, niños y adolescentes víctimas de comercio sexual". Tesis de licenciatura. Universidad de Costa Rica, San José, C.R. 2006.

Blazquez Graf, Norma. 2012. "Epistemología feminista: temas centrales”. En: Investigación feminista: epistemología, metodología y representaciones sociales. Coordinador por: Blazquez, Norma, Flores, Fátima y Ríos, Maribel. México: UNAM.

Calderón, Maribelle y Vargas, Sonia. "Construcción subjetiva de la masculinidad hegemónica en dos grupos de hombres mayores de edad, que han experimentado o no violencia intrafamiliar en sus hogares de origen: su relación con el ejercicio de poder y control con su pareja”. Tesis de licenciatura. Universidad de Costa Rica, San José, C.R. 2004

Calderón Gómez, Marianela. "La madre ante la situación de incesto: sus vivencias, conflictos y necesidades en la provincia de Guanacaste: un estudio exploratorio". Tesis de licenciatura. Universidad de Costa Rica, San José, C.R. 2003.

Carvajal Oirich, Zaira. "Prevalencia, manifestaciones y efectos del hostigamiento sexual en la Universidad Nacional”. Tesis de maestría. Universidad de Costa Rica, San José, C.R. 2004

Castro, Dyalá y Chávez, Karla. "Explotación sexual comercial y el ciclo intergeneracional de la violencia basada en género: estudio de casos a partir del modelo contextual intergeneracional”. Tesis de licenciatura. Universidad de Costa Rica, San José, C.R. 2002.

Castro Chaves, Xiomara. "Equidad de género en el noviazgo: guía didáctica dirigida a adolescentes mujeres”. Tesis de licenciatura. Universidad de Costa Rica, San José, C.R. 2012. 
Chacón, Laura y Picado, Helen. "Características psicológicas y sociodemográficas en mujeres adultas sobrevivientes de incesto infanto-juvenil del Área Metropolitana". Tesis de licenciatura. Universidad de Costa Rica, San José, C.R. 2005.

Claramunt Montero, María Cecilia. 1997. Casitas Quebradas: El problema de la violencia doméstica en Costa Rica. San José: EUNED.

Claramunt Montero, Maria Cecilia. "Explotación sexual comercial y trabajo infantil. Análisis de la respuesta estatal costarricense a las personas menores de edad y sus familias desde el enfoque de los derechos humanos". Tesis de maestría. Universidad de Costa Rica, San José, C.R. 2005.

Cordero, Michelle. "Mujeres cristianas practicantes: Relación entre la doctrina-praxis de sus comunidades religiosas y su vivencia de violencia doméstica”. Tesis de licenciatura. Universidad de Costa Rica, San José, C.R. 2003.

Cover, Jeannette. "Prevalencia del abuso sexual infantil en poblaciones universitarias". Tesis de licenciatura. Universidad de Costa Rica, San José, C.R. 1995.

De Miguel, Ana. 2015. Neoliberalismo sexual. El mito de la libre elección. España: Ediciones Cátedra, Universitat de Valencia.

Díaz, Katalina y Navarrete, Rebeca. "El disfrute de la sexualidad en algunas mujeres adultas sobrevivientes de abuso sexual infantil, de la provincia de Guanacaste". Tesis de licenciatura”. Universidad de Costa Rica, Guanacaste, C.R. 2005.

Fernández, Mayra. “Análisis psicosocial del ciclo de agresión contra la mujer dentro del vínculo de la relación conyugal. Realizado en la provincia de Puntarenas con un grupo de mujeres del sector popular”. Tesis de licenciatura. Universidad de Costa Rica, San José, C.R. 1996.

González, Karla. "El proceso de resignificación de la experiencia en casos de violencia conyugal contra la mujer: un estudio desde el modelo de resiliencia”. Tesis de licenciatura. Universidad de Costa Rica, San José, C.R. 2001.

Hernández Vega, Melissa. Impacto psicosocial de la violencia obstétrica a través de relatos de vida de mujeres. Tesis de licenciatura. Universidad de Costa Rica, San José, C.R. 2020. 
Incer Brenes, Graciela. "Por qué se van las mujeres: factores que impulsan a las mujeres a terminar o rechazar relaciones de noviazgo en las que hay violencia, la historia de tres mujeres”. Tesis de licenciatura. Universidad de Costa Rica, San José, C.R. 2009.

Instituto Nacional de las Mujeres. 2019. Tercer Estado de los Derechos Humanos de las Mujeres en Costa Rica. San José: INAMU.

Jayme, María y Sau, Victoria. 2004. Psicología diferencial del sexo. Barcelona: ICARIA.

Loaiza Durán, Magaly. "Con rostro femenino: la violencia por motivos de género en contra de las mujeres y la transmisión del VIH”. Tesis de maestría. Universidad de Costa Rica, Sn José, C.R. 2008.

Marín, Ingrid. "Prevalencia y manifestaciones del hostigamiento sexual en el Instituto Nacional de Aprendizaje INA”. Tesis de maestría. Universidad de Costa Rica, San José, C.R. 2012.

Marín, Ileana y Villalobos, Vanessa. "Violencia doméstica: un análisis psicosocial sobre la perspectiva del hombre privado de libertad que golpea a su pareja”. Tesis de licenciatura. Universidad de Costa Rica, San José, C.R. 1999.

Mesén Badilla, Amanda. "Acoso sexual en espacios públicos. "Análisis narrativo de las experiencias vivenciadas por mujeres y publicadas en la red social Facebook". Tesis de licenciatura. Universidad de Costa Rica, San José, C.R. 2019.

Molina Lobo, Marta y Moreira Chavarría, Ruthman. "Representaciones sociales acerca del abuso sexual infantil en niños y niñas de una escuela de la zona del Pacífico Central”. Tesis de licenciatura. Universidad de Costa Rica, San José, C.R. 2006.

Murillo, María Elena. "Reflexión con adolescentes en torno a formas de violencia en sus relaciones de noviazgo. Un estudio exploratorio con adolescentes de 16 a 18 años, de dos colegios públicos del Área Metropolitana de San José”. Tesis de licenciatura. Universidad de Costa Rica, San José, C.R. 1996.

Pacheco, Adrián. "Violencia contra las mujeres por parte de sus cónyuges. Un modelo confirmatorio desde el enfoque de género y la psicología evolucionaria”. Tesis de maestría. Universidad de Costa Rica, San José, C.R. 2009.

Pisoni, Mariana."La psicopatía como factor de riesgo para la realización de la ofensa sexual en población juvenil masculina costarricense". Tesis de licenciatura. Universidad de Costa Rica, San José, C.R. 2006. 
Ramírez, Isela. "Violencia doméstica: La mujer agredida. La problemática desde su punto de vista”. Tesis de licenciatura. Universidad de Costa Rica, San José, C.R.1997.

Retana Arguedas, Fresia. "El concepto del cuerpo en mujeres abusadas sexualmente: un análisis psico-social”. Tesis de licenciatura. Universidad de Costa Rica, San José. C.R. 1998.

Rodríguez Fernández, Adriana. "Vivencia y construcción de la maternidad en niñas y adolescentes vinculadas a la explotación sexual comercial”. Tesis de licenciatura. Universidad de Costa Rica, San José, C.R. 2006.

Rodríguez, Adriana y Rovinski, Mijal. 2019. “A dos décadas de la Convención de Belem do Pará: Una caracterización de la investigación psicológica sobre la violencia contra las mujeres". Revista Wimblu, 14(2). Recuperado de https://revistas.ucr.ac.cr/index.php/wimblu/article/view/39600/40160

Rodríguez Pizarro, Nubia e Ibarra Melo, María Eugenia. 2013. "Los estudios de género en Colombia. Una discusión preliminar." Revista Sociedad y Economía: 24: 15-46. Recuperado de: http://www.redalyc.org/articulo.oa?id=99629534002

Rojas, Silvia y García, Alejandra. "Abuso emocional en el ámbito educativo costarricense: Desde la experiencia de un grupo de hombres y mujeres adolescentes y sus docentes". Tesis de licenciatura. Universidad de Costa Rica, San José, C.R. 2003.

Salas Torres, Maricel. "Los significados psicosociales del acoso sexual a las mujeres en el ambiente de trabajo: el caso del ICE”. Tesis de maestría. Universidad de Costa Rica, San José, C.R. 1996.

Salas Torres, Maricel. "El acoso sexual en el trabajo: significados en los albores del nuevo milenio”. Tesis de licenciatura. Universidad de Costa Rica, San José, C.R. 2002

Sandí, Vileka y Venegas, Luis Alonso. "Un estudio casuístico de los celos en hombres que han ejercido violencia intrafamiliar". Tesis de licenciatura. Universidad de Costa Rica, San José, C.R. 2008.

Segura, Gabriela. "El cuerpo habitado: Un análisis de la representación de su cuerpo en mujeres adolescentes relacionadas con la prostitución”. Tesis de licenciatura. Universidad de Costa Rica, San José, C.R. 1999. 
Sequeira, Alejandra y Vázquez, Marcela. "Psicopatología y funcionamiento ejecutivo en mujeres que han experimentado violencia en sus relaciones de pareja". Tesis de licenciatura. Universidad de Costa Rica, San José, C.R. 2015.

Torres Campos, Taryn. "El hostigamiento sexual en un centro educativo de la ciudad de Liberia: la experiencia de dos mujeres adolescentes que denunciaron a un profesor en el 2006”. Tesis de licenciatura. Universidad de Costa Rica, San José, C.R. 2009.

Vargas Cascante, María de los Ángeles. "Mirando en retrospectiva: consecuencias cognitivas, conductuales y emocionales en mujeres adultas mayor que presenciaron violencia contra su madre y que, a su vez, sufren violencia, visto desde el enfoque de género". Tesis de licenciatura. Universidad de Costa Rica, San José, C.R. Rodrigo Facio. 2012.

Vega, Melissa. "Guía didáctica dirigida a proveedores y proveedoras de servicios de salud para la atención primaria de mujeres embarazadas en situación de violencia doméstica”. Tesis de licenciatura. Universidad de Costa Rica, San José, C.R. 2009.

Villalobos, Ana Marcela y Naranjo, Soledad. Incesto en mujeres adolescentes: Características psicológicas y sociales. Un estudio de casos (Tesis de licenciatura). Universidad de Costa Rica, San José, C.R. 1994. 